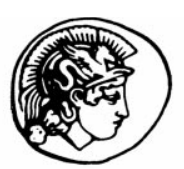

PERGAMON

Computers \& Fluids 28 (1999) 779-799

\title{
A study on the high-order Smolarkiewicz methods
}

\author{
Hung-Chi Kuo ${ }^{\text {a }}$, Tzay-Ming Leou ${ }^{\mathrm{b}}$, R.T. Williams ${ }^{\mathrm{c}, *}$ \\ ${ }^{a}$ Department of Meteorology, Naval Postgraduate School, 589 Dyer Road, Bldg. 235, Rm 254, Monterey, \\ CA 93943-5114, USA \\ ${ }^{\mathrm{b}}$ Computer Center, Central Weather Bureau, Taipei, Taiwan, ROC \\ ${ }^{\mathrm{c}}$ Department of Meteorology, Naval Postgraduate School, Monterey, CA, USA
}

Received 22 August 1997; received in revised form 6 July 1998; accepted 21 July 1998

\begin{abstract}
A modified Smolarkiewicz positive definite scheme is proposed. The computational cost of the proposed scheme is exactly the same as the high-order (third-order accurate) Smolarkiewicz method. We test numerically the convergence rate, stability, accuracy, peak value maintenance and mass loss for the method. Comparative studies of the modified scheme with other positive definite schemes, the fourthorder finite difference and the semi-Lagrangian method are performed. It is found that the modified scheme provides an error convergence rate which is comparable to both the semi-Lagrangian and fourth-order finite difference schemes. When the advective field is smooth enough, the modified scheme yields improved fourth-order accuracy in the low Courant number situation. When the Courant number is larger than 0.5, the modified procedure yields the same result as the third-order Smolarkiewicz scheme. Other advection calculations with a slotted cylinder yield results which compare favorably with the results of the high-order (third-order accurate) Smolarkiewicz method and the semi-Lagrangian method. (C) 1999 Elsevier Science Ltd. All rights reserved.
\end{abstract}

\section{Introduction}

The conservation equation for the density $\psi$ of a substance advected with a fluid can be written as

$$
\frac{\partial \psi}{\partial t}+\nabla \cdot(\psi \mathbf{V})=\mathbf{S}
$$

with the sink or source term of $S$ on the right hand side. There are many applications of

\footnotetext{
*Corresponding author. Tel.: + 1-831-656-3788; Fax.: +1-831-656-3061. 
equations like Eq. (1) in meteorology, oceanography and other fields. This involves the conservation of some positive-definite variables such as potential vorticity, chemical tracers and total airborne moisture density. A general complaint with the traditional numerical solutions of Eq. (1), regardless of whether the discretization is spectral, finite difference or finite element, is that they usually lead to negative values in the positive-definite fields. In addition, the schemes cause the spurious numerical over- and undershots in regions of steep gradients. The problem is most noticeable as regions of negative mixing ratio which is inconsistent with physical parameterization and reality. Various computational fixes have been included in models for the negative regions [1]. However, they result in significant computational sources or computational transport.

When faced with the prospect of choosing a positive definite advection scheme, the scientist confronts a very large number of methods. Low-order schemes such as upstream advection produce no ripples but suffer from excessive implicit diffusion. On the other hand, methods like the flux-corrected transport (FCT) developed by Boris and Book [2] and generalized by Zalesak [3], are appropriate to maintain a sharp gradient (such as shock wave) without producing ripples. However, these methods can also quickly fracture a smooth solution into a series of steps. The schemes also require significant computer time [1]. Tremback et al. [4] extended the forward-in-time upstream advection scheme to higher orders. Hsu and Arakawa [5] proposed a generalization of Takacs' [6] third-order finite difference scheme that has very small dissipation and computational dispersion, and that guarantees positive definiteness in the time continuous situation. Hsu and Arakawa [5] used the method to simulate baroclinic instability with an isentropic vertical coordinate. Williamson and Rasch [7] used shape-preserving interpolation in the semi-Lagrangian method to maintain the local monotonicity of the moisture fields in a global model. Bott [8-10] presented an "area preserving" positive definite advection scheme obtained by nonlinear renormalization of the advective fluxes. Recently, Holnicki [11] put forward a shape-preserving interpolation in the semi-Lagrangian advection.

Smolarkiewicz $[12,13]$ used Taylor expansion derived antidiffusions to counter the excessive diffusion associated with the upstream advection method. Depending on the order of antidiffusion, the Smolarkiewicz family of positive definite schemes is very efficient and accurate. They have been applied successfully to the cumulus cloud-topped entrainment simulations and many other cloud modeling problems. Smolarkiewicz and Clark [14], Smolarkiewicz and Grabowski [15] and Smolarkiewicz and Rasch [16] provided further developments and applications of the technique. More recently, Margolin and Smolarkiewicz [17] give an adequate description of the genuine third-order multidimensional positive definite advection transport algorithm. In this paper, we propose a modified high-order Smolarkiewicz scheme which is fourth-order accurate in low Courant number computations. We also compare the high-order Smolarkiewicz scheme with other positive definite schemes, the fourth-order finite difference and the semi-Lagrangian method. Section 2 gives the general family of the Smolarkiewicz methods as well as the proposed modification. The stability and consistency conditions for the proposed method are also discussed in Section 2. The numerical results for solid body rotation with a Gaussian cone and a slotted cylinder are presented in Section 3. Section 4 gives the concluding remarks. 


\section{The Smolarkiewicz methods}

\subsection{Low- and high-order Smolarkiewicz schemes}

Details of the Smolarkiewicz positive definite method can be found in $[12,13]$. The first stage of the method is to apply the upstream advection scheme to the values at $n \Delta t$ level on a stagger grid (e.g. $\psi_{i}$ and $u_{i+1 / 2}$ for $1 \leq i \leq I$ )

$$
\frac{\psi_{i}^{(0)}-\psi_{i}^{n}}{\Delta t}=-\frac{F_{i+1 / 2}^{n}-F_{i-1 / 2}^{n}}{\Delta x}
$$

where

$$
F_{i+1 / 2}^{n}=\frac{1}{2}\left(\left(u_{i+1 / 2}^{n}+\left|u_{i+1 / 2}^{n}\right|\right) \psi_{i}^{n}+\left(u_{i+1 / 2}^{n}-\left|u_{i+1 / 2}^{n}\right|\right) \psi_{i+1}^{n}\right)
$$

The strong implicit diffusion associated with the upstream advection can be determined by Taylor expansion methods. The fundamental idea of the Smolarkiewicz methods lies in performing the antidiffusion calculations in the upstream fashion. In the case of uniform flow, expanding the upstream calculated $\psi_{i \pm 1}^{(0)}$ (newly predicted $\psi$ at $(i \pm 1)$ grids) in a third-order in the space and time Taylor sum about $\left(x_{i}, n \Delta t\right)$, we have

$$
\left.\left(\frac{\partial \psi}{\partial t}+\frac{\partial u \psi}{\partial x}\right)\right|_{i} ^{n}=\left.\frac{\partial}{\partial x}\left[\frac{1}{2}\left(|u| \Delta x-u^{2} \Delta t\right) \frac{\partial \psi}{\partial x}-\frac{1}{6}\left(u \Delta x^{2}-u^{3} \Delta t^{2}\right) \frac{\partial^{2} \psi}{\partial x^{2}}\right]\right|_{i} ^{n}+O\left(\Delta t^{3}, \Delta x^{3}\right)
$$

The right hand side of Eq. (3) is the implicit diffusion associated with the upstream advection. To antidiffuse the implicit diffusion associated with upstream advection, we reverse the effect of diffusion by defining an 'antidiffusion velocity' [12] and [14]

$$
\tilde{u}=\frac{1}{2} \frac{\left(|u| \Delta x-u^{2} \Delta t\right) \partial \psi / \partial x}{\psi}-\frac{1}{6} \frac{\left(u \Delta x^{2}-4 u^{3} \Delta t^{2}+3 u|u| \Delta x \Delta t\right) \partial^{2} \psi / \partial x^{2}}{\psi}+O\left(\Delta t^{3}, \Delta x^{3}\right)
$$

for the reversal diffusion. Namely, we compute the equation

$$
\frac{\partial \psi}{\partial t}=\frac{\partial \tilde{u} \psi}{\partial x}
$$

in the upstream discretization for the antidiffusion. The process of antidiffusion can be repeated until the desired result is reached.

Note that the first term on the right-hand side of Eq. (4) which involves $\Delta x$ and $\Delta t$ stems from second-order in the space and time Taylor expansions. Similarly, the second term on the right hand side of Eq. (4) stems from the third-order in the space and time Taylor expansions. Depending on the order of the Taylor expansion to be included in antidiffusion, we have the Smolarkiewicz family of schemes $[12,13]$. Without the second term in Eq. (4), we have the low (second-order accurate) order Smolarkiewicz scheme [12]. We obtain the high-order (thirdorder accurate) Smolarkiewicz scheme $[13,17]$ by retaining both terms on the right hand side of Eq. (4). The antidiffusion process in the Smolarkiewicz schemes can be written as an iterative upstream scheme with a total of $M$ iterations. 
The discrete equation for the antidiffusion is

$$
\frac{\psi_{i}^{(m+1)}-\psi_{i}^{(m)}}{\Delta}=\frac{\tilde{F}_{i+1 / 2}^{(m)}-\tilde{F}_{i-1 / 2}^{(m)}}{\Delta \mathrm{x}}
$$

where

$$
F_{i+1 / 2}^{(m)}=\frac{\left(\tilde{u}_{i+1 / 2}^{(m)}+\left|\tilde{u}_{i+1 / 2}^{(m)}\right|\right)}{2.0} \psi_{i}^{(m)}+\frac{\left(\tilde{u}_{i+1 / 2}^{(m)}-\left|\tilde{u}_{i+1 / 2}^{(m)}\right|\right)}{2.0} \psi_{i+1}^{(m)}
$$

for $1 \leq m \leq M$. Starting with Eq. (2), we obtain the $\psi$ values at $(n+1) \Delta t$ after $M$ iterations of Eq. (6).

Depending on the choices of $\tilde{u}$ in Eq. (6), we have the Smolarkiewicz family of schemes. Two Smolarkiewicz schemes are considered. The first one is the second-order accurate Smolarkiewicz scheme

$$
\tilde{u}_{i+1 / 2}^{(m)}=\frac{\left(\left|\tilde{u}_{i+1 / 2}^{(m-1)}\right| \Delta x-\left(\tilde{u}_{i+1 / 2}^{(m-1)}\right)^{2} \Delta t\right)\left(\psi_{i+1}^{(m-1)}-\psi_{i}^{(m-1)}\right)}{\Delta x\left(\psi_{i+1}^{(m-1)}+\psi_{i}^{(m-1)}+\epsilon\right)}
$$

The second one is the third-order accurate Smolarkiewicz scheme

$$
\begin{aligned}
\tilde{u}_{i+1 / 2}^{(m)}= & \frac{\left(\left|\tilde{u}_{i+1 / 2}^{(m-1)}\right| \Delta x-\left(\tilde{u}_{i+1 / 2}^{(m-1)}\right)^{2} \Delta t\right)\left(\psi_{i+1}^{(m-1)}-\psi_{i}^{(m-1)}\right.}{\Delta x\left(\psi_{i+1}^{(m-1)}+\psi_{i}^{(m-1)}+\epsilon\right)}-\frac{1}{3}\left(\tilde{u}_{i+1 / 2}^{(m-1)} \Delta x^{2}-4\left(\tilde{u}_{i+1 / 2}^{(m-1)}\right)^{3} \Delta t^{2}\right. \\
& \left.+3 \tilde{u}_{i+1 / 2}^{(m-1)}\left|\tilde{u}_{i+1 / 2}^{(m-1)}\right| \Delta x \Delta t\right) \times \frac{\left(\psi_{i+2}^{(m-1)}+\psi_{i-1}^{(m-1)}-\psi_{i+1}^{(m-1)}-\psi_{i}^{(m-1)}\right)}{\Delta x^{2}\left(\psi_{i+2}^{(m-1)}+\psi_{i-1}^{(m-1)}+\psi_{i+1}^{(m-1)}+\psi_{i}^{(m-1)}+\epsilon\right)}
\end{aligned}
$$

The $\epsilon$ in Eqs. (7) and (8) is a small value, e.g. $10^{-15}$ to ensure $\tilde{u}^{(m)}=0$ when $\psi_{i \pm 1}^{(m-1)}=\psi_{i+2}^{(m-1)}=\psi_{i}^{(m-1)}=0$. The values of $\psi_{i}^{(0)}$ for the $m=1$ situation in either Eq. (7) or (8) are taken from Eq. (2). Similarly, the $\tilde{u}_{i}^{(0)}{ }_{1 / 2}$ required in either Eq. (7) or (8) are taken from $u_{i}^{n}+1 / 2$.

In summary, Eqs. (2), (6) and (7) define the low order Smolarkiewicz scheme. The number of iterative antidiffusions is optional. Similarly, Eqs. (2), (6) and (8) define the high-order Smolarkiewicz scheme. They differ from each other only in the order of accuracy in the 'antidiffusion velocity'. The multidimensional advection in the Smolarkiewicz method can be generalized by the time splitting technique [12]. We have employed the time-splitting technique in this paper.

\subsection{The modified Smolarkiewicz method}

With a slight modification in computing the antidiffusion velocity, we have our proposed scheme. The modified Smolarkiewicz method is the same as the high-order Smolarkiewicz method up to the first antidiffusion $(m=1)$ iteration. Starting from the second iteration of antidiffusion $(2 \leq m \leq M)$, we use the computed low order antidiffusion velocity ( first term on 
the right hand side of Eq. (8)) to evaluate the high-order antidiffusion term (second term on the right hand side of Eq. (8)). Thus, our modified version has the same computational counts as the high-order Smolarkiewicz method.

The $\tilde{u}^{(m)}$ of the proposed method is

$$
\begin{aligned}
\tilde{u}_{i+1 / 2}^{(1)}= & \frac{\left(\left|\tilde{u}_{i+1 / 2}^{(0)}\right| \Delta x-\left(\tilde{u}_{i+1 / 2}^{(0)}\right)^{2} \Delta t\right)\left(\psi_{i+1}^{(0)}-\psi_{i}^{(0)}\right)}{\Delta x\left(\psi_{i+1}^{(0)}+\psi_{i}^{(0)}+\epsilon\right)}-\frac{1}{3}\left(\tilde{u}_{i+1 / 2}^{(0)} \Delta x^{2}-\left(\tilde{u}_{i+1 / 2}^{(0)}\right)^{3} \Delta t^{2}\right) \\
& \times \frac{\left(\psi_{i+2}^{(0)}+\psi_{i-1}^{(0)}-\psi_{i+1}^{(0)}-\psi_{i}^{(0)}\right)}{\Delta x^{2}\left(\psi_{i+2}^{(0)}+\psi_{i-1}^{(0)}+\psi_{i+1}^{(0)}+\psi_{i}^{(0)}+\epsilon\right)}
\end{aligned}
$$

and

$$
\begin{aligned}
\tilde{u}_{i+1 / 2}^{(*)} & =\frac{\left(\left|\tilde{u}_{i+1 / 2}^{(m-1)}\right| \Delta x-\left(\tilde{u}_{i+1 / 2}^{(m-1)}\right)^{2} \Delta t\right)\left(\psi_{i+1}^{(m-1)}-\psi_{i}^{(m-1)}\right)}{\Delta x\left(\psi_{i+1}^{(m-1)}+\psi_{i}^{(m-1)}+\epsilon\right)} \\
\tilde{u}_{i+1 / 2}^{(m)} & =\tilde{u}_{i+1 / 2}^{(*)}-\frac{1}{3}\left(\tilde{u}_{i+1 / 2}^{(*)} \Delta x^{2}-4\left(\tilde{u}_{i+1 / 2}^{(*)}\right)^{3} \Delta t^{2}+3 \tilde{u}_{i+1 / 2}^{(*)}\left|\tilde{u}_{i+1 / 2}^{(*)}\right| \Delta x \Delta t\right) \\
& \times \frac{\left(\psi_{i+2}^{(m-1)}+\psi_{i-1}^{(m-1)}-\psi_{i+1}^{(m-1)}-\psi_{i}^{(m-1)}\right)}{\Delta x^{2}\left(\psi_{i+2}^{(m-1)}+\psi_{i-1}^{(m-1)}+\psi_{i+1}^{(m-1)}+\psi_{i}^{(m-1)}+\epsilon\right)}
\end{aligned}
$$

for $2 \leq m \leq M$.

Eqs. (2), (6) and (9) constitute our proposed method. The idea here is similar to using newly updated values in the Gauss-Seidel method to improve over the Jacobi method for iterative solutions. However, the nonlinear nature of the method makes the proof very difficult.

\subsection{Stability and consistency conditions of the modified Smolarkiewicz method}

The proof of the consistency condition in the Smolarkiewicz family of methods can be found in $[12,13]$. To prove consistency for our modified scheme it is enough to show that when $\Delta t$, $\Delta x$ approach 0, Eq. (6) with Eq. (9)b) ( $m \geq 2)$ does not affect the solution computed from Eq. (6) with Eq. (9)a) $(m=1)$. The stability condition of the Smolarkiewicz method takes the form

$$
\max _{i}\left(\frac{\left|\tilde{u}_{i+1 / 2}^{(m)}\right| \Delta t}{\Delta x}\right) \leq 1
$$

which is the same as the upstream method. When $\Delta x$ and $\Delta t$ approach $0, \psi_{i+}^{(m)}{ }_{1}-\psi_{i}^{(m)}$ will approach 0. With Eq. (10)a), it is easy to see that $\tilde{u}_{i+1 / 2}^{(*)}$ and thus $\tilde{u}_{i+1 / 2}^{(m)}$ will also approach 0 in Eq. (9)b). Thus Eq. (6) with the antidiffusion velocity Eq. (9)b), approaches the form 


$$
\frac{\partial \psi^{(m)}}{\partial t}=0
$$

which means that the proposed scheme is consistent.

To demonstrate stability of the proposed scheme it is enough to show that stability of Eq. (6) with Eq. (9a) $(m=1)$ implies the stability of Eq. (6) with Eq. (9b) $(m \geq 2)$. Because the upstream scheme is used, the stability condition for $m \geq 2$ takes the form of Eq. (10a) with the $\tilde{u}_{i+1 / 2}^{(m)}$ defined by either Eq. (9a) or (9b). Substituting Eq. (9b) in the condition (10a), and defining

$$
\begin{aligned}
A_{i} & =\frac{\left|\psi_{i+1}^{(m-1)}-\psi_{i}^{(m-1)}\right|}{\left(\psi_{i+1}^{(m-1)}+\psi_{i}^{(m-1)}+\epsilon\right)} \\
B_{i} & =\frac{\left|\left(\psi_{i+2}^{(m-1)}+\psi_{i-1}^{(m-1)}-\psi_{i+1}^{(m-1)}-\psi^{(m-1)}\right)\right|}{\left(\psi_{i+2}^{(m-1)}+\psi_{i-1}^{(m-1)}+\psi_{i+1}^{(m-1)}+\psi_{i}^{(m-1)}+\epsilon\right)} \\
C_{i} & =\left(\frac{\left|\tilde{u}_{i+1 / 2}^{(m-1)}\right| \Delta t}{\Delta x}\right)\left(1-\left[\frac{\left|\tilde{u}_{i+1 / 2}^{(m-1)}\right| \Delta t}{\Delta x}\right]\right)
\end{aligned}
$$

we have

$$
\max _{i}\left(\frac{1}{3} C_{i} A_{i} B_{i}\left(1-C_{i}^{2} A_{i}^{2}\right)\right) \leq 1
$$

The inequality in Eq. (14) stems from the fact that the maximum value of $C_{i}$ is 0.25 [12] and the terms $A_{i}$ and $B_{i}$ are less than or equal to unity from Eq. (10a). As discussed in [12], the maximum of $C_{i}=0.25$ allows values of $\tilde{u}$ to increase without destroying the stability of the scheme.

\section{Numerical results}

We employ the first-order accurate upstream scheme (hereafter $U P$ ), third-order accurate Hsu and Arakawa (hereafter $H A$ ), fourth-order finite difference (hereafter $F D 4$ ), second-order Smolarkiewicz method with two iterations of antidiffusion (hereafter $S M L 2$ ), third-order Smolarkiewicz method with three iterations of antidiffusion (hereafter SMH3), semiLagrangian with cubic spline interpolation (hereafter $S L$ ) and the proposed modified Smolarkiewicz method with three and four iterations of antidiffusion (hereafter MSM3 and MSM4, respectively) in our numerical calculation.

We consider the solid body rotation

$$
\frac{\partial \psi}{\partial t}+\frac{\partial U \psi}{\partial x}+\frac{\partial V \psi}{\partial y}=0
$$

in the closed domain $[-1,1] \times[-1,1]$, with $U=-\omega y$ and $V=\omega x$ and the $\omega=2 \pi / 9$. 
A Gaussian cone is used as the initial condition for Eq. (13) so that

$$
\psi(x, y, 0)=\exp \left[-\left(\frac{x+0.3}{0.2}\right)^{2}-\left(\frac{y+0.3}{0.2}\right)^{2}\right]
$$

To substantiate the difference between the SMH3 method and the MSM3 method, a complete convergence path test in terms of $\Delta x$ and $\Delta t$ is performed. Fig. 1 gives the logarithm of the root-mean-square error in the numerical solutions of the solid body rotation with Gaussian cone rotation as function of $N$ (number of grid points) and Courant number $(\Delta t / \Delta x)$. Fig. 1 indicates that our modified MSM3 leads to improvement in accuracy when the Courant number is smaller than 0.5. The MSM3 method yields results similar to the $S M H 3$ method when the Courant number is greater than 0.5. In view that the time step in meteorological models without the semi-implicit method is limited by the stability constraint rather than by the accuracy constraint, the improvement in accuracy in MSM3 in the low Courant number situation may be significant. In the following comparisons, we will concentrate only on low Courant number calculations.

We employ $\Delta t=1 / 160$ and $N=32$ for both $x$ and $y$ directions in our test. Thus, it takes 1440 time steps for the cone to rotate back to the initial position. The Gaussian function is used because it is infinitely differentiable and is well suited for the convergence rate test. Fig. 2 gives the numerical solutions after one circulation of the solid body rotation with the Gaussian cone. It is apparent that the $H A$ and $S M L 2$ yield a peak value smaller than the peak values that result from the $S M H 3, F D 4$ and $M S M 4$ methods. Except for the spurious negative values, the FD4 gives a better approximation to the advection problem than the $S M L 2$ and $H A$ methods. The $S M H 3$ and MSM3 methods yield results similar to the $F D 4$, method but without the negative values. Finally, we note that the high-order Smolarkiewicz methods yield results that compare favorably with the semi-Lagrangian method.

Because of the nonlinear nature of the MSM3 and MSM4 methods, the order of the accuracy cannot be determined by Fourier analysis. Instead, the convergence rate of the methods will be determined by the computational errors as a function of $N$. Fig. $3 a$ gives the root-mean-square errors in numerical solutions of the test problem (13) as a function of $N$ for the UP, SML2, HA, SMH3 and FD4 methods after one circulation of the Gaussian cone. The first-order convergence rate of the UP method, second order of the SML2 method, third-order of the $H A$ and $S M H 3$ methods and fourth-order of the FD4 method can be seen from the slopes of the curves. Fig. $3 \mathrm{~b}$ is similar to Fig. 3a except for the MSM3, MSM4 and $S L$ methods. The errors of FD4 and $H A$ are included for the comparison. The MSM4 scheme converges slightly faster than the MSM3 scheme. It is clear from the slopes of the curves in Fig. $3 \mathrm{~b}$ that the proposed modified Smolarkiewicz methods (MSM3 and MSM4) are fourthorder accurate in this low Courant number situation.

Fig. 4a gives the peak value in the numerical solutions as a function of $N$ for the $U P$, SML2, HA, SMH3 and FD4 methods. Clearly, the SMH3 method yields results which compare favorably with the FD4 method. Moreover, it takes about $N=24$ (about $2 \Delta x$ for the $e$-folding distance of the advected Gaussian cone) for both the FD4 and $S M H 3$ methods to have peak values close to 1 . Fig. $4 \mathrm{~b}$ is similar to Fig. 4a except for the $M S M 3, M S M 4$ and $S L$ methods. The $H A$ method is included for the comparison. It is concluded from Fig. $4 \mathrm{~b}$ that the 
(a) $\mathrm{SMH3}$

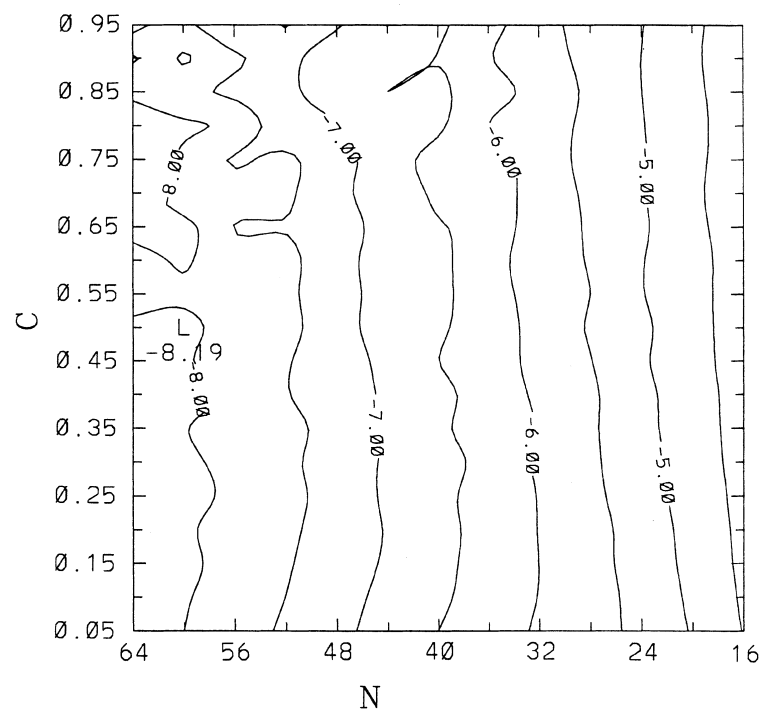

(b) MSM3

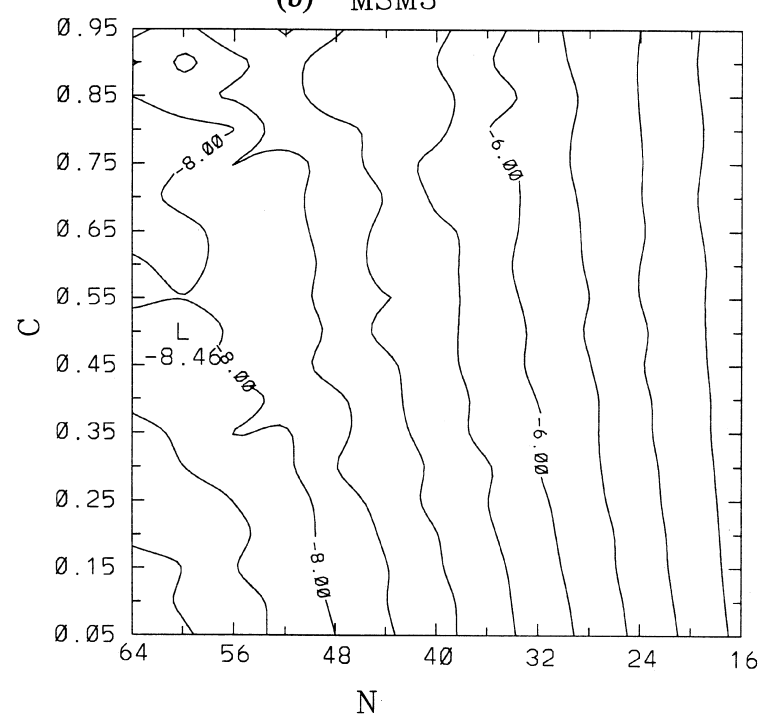

Fig. 1. The logarithm of the root-mean-square error in the numerical solutions of the solid body rotation with Gaussian cone rotation as function of $N$ (the number of grids) and Courant number $(\Delta t / \Delta x)$ for (a) the $S M H 3$ methods and (b) the MSM3 method.

MSM3, MSM4 and SL methods possess similar peak values. After careful comparison with the $S M H 3$ and MSM3 methods, we see that the peak value associated with the MSM3 method is closer to 1 . Our experience also indicates that it is not necessary to use the four iterations in the proposed modified scheme.

Finally, we test the time-step stability of the above methods. Fig. 5a gives the root-meansquare error as a function of time step size $\left(\ln _{2} \Delta t\right)$ for the $H A, S M L 2$ and $S M H 3$ methods 
after one circulation of cone rotation when $N=32$. Fig. $5 \mathrm{~b}$ is similar to Fig. 5a except for the FD4, MSM3 and MSM4 methods. When the time step is small, the nearly flat lines reflect the spatial discretization error of the methods. Fig. 5 shows that the FD4 method has a more restricted stability constraint than the other methods. The family of Smolarkiewicz methods have a similar time stability constraint which agrees with the analysis in Section 2c. Our experience indicates that the SMH3, MSM3 and $H A$ methods take about the same
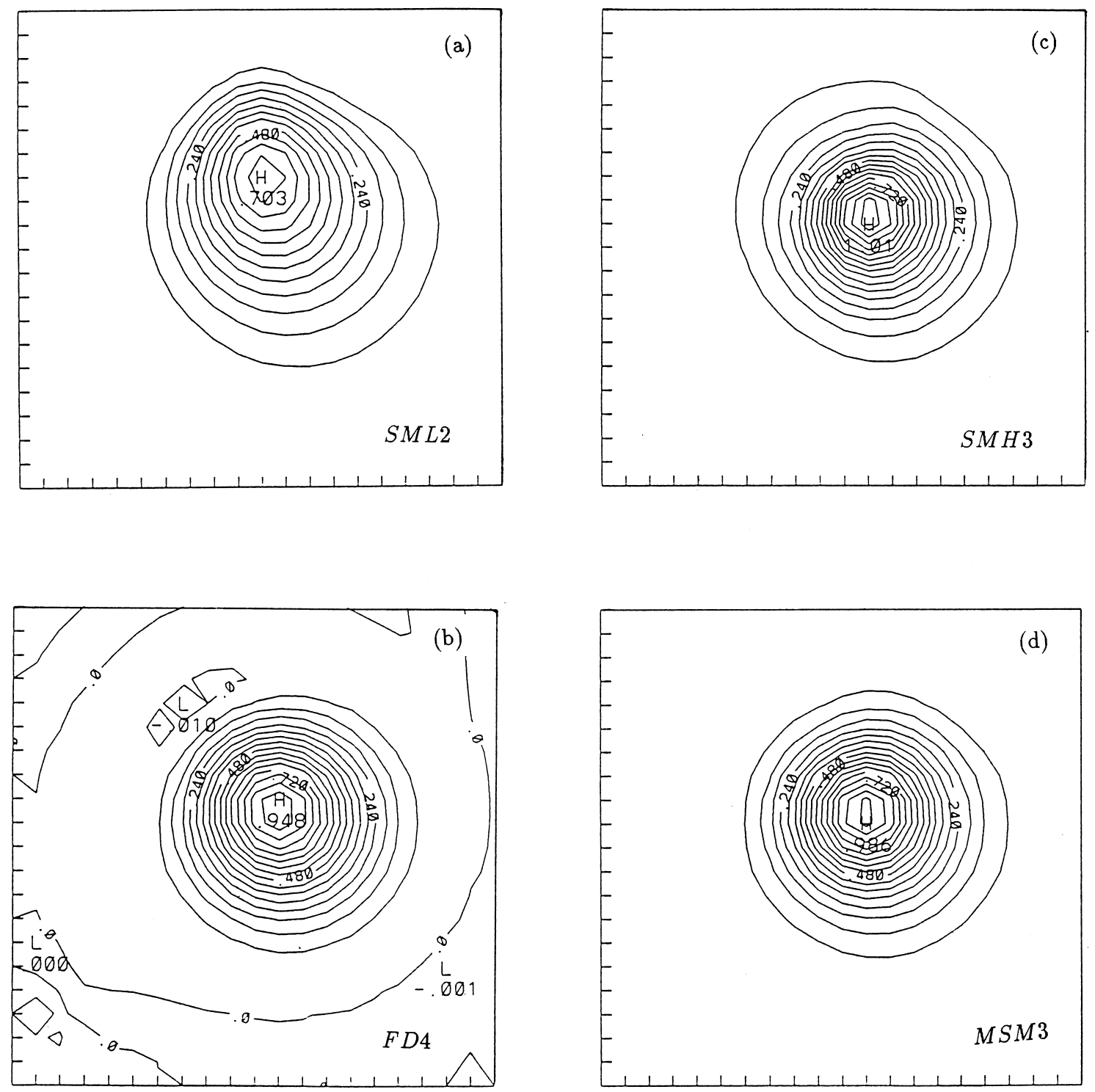

Fig. 2. Numerical solutions of the solid body rotation with Gaussian cone rotation after one circulation with $N=32$ for (a) $S M L 2$ method, (b) FD4 method, (c) $S M H 3$ method, (d) MSM3 method, (e) $H A$ method and (f) $S L$ method. 

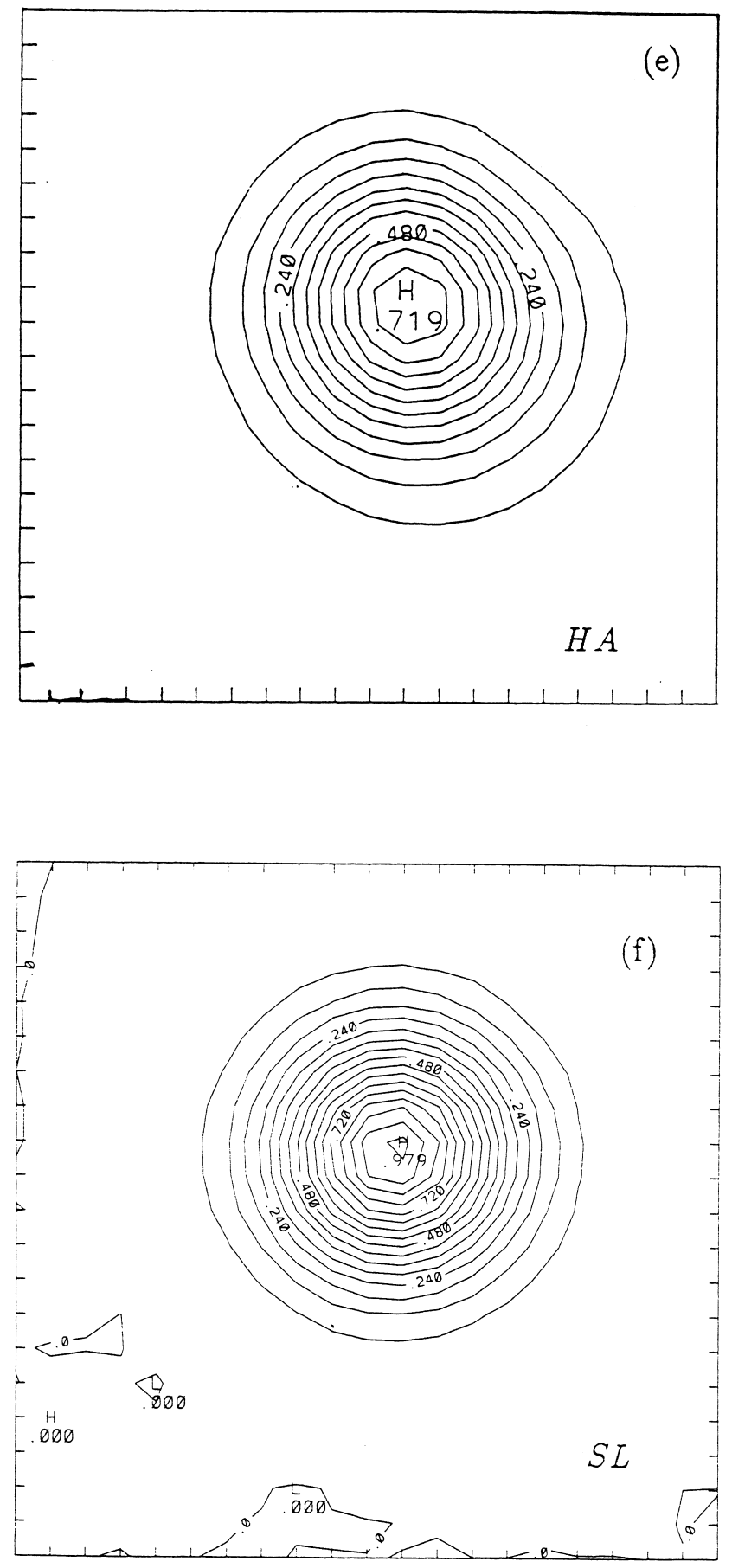

Fig. 2 (e, f) 

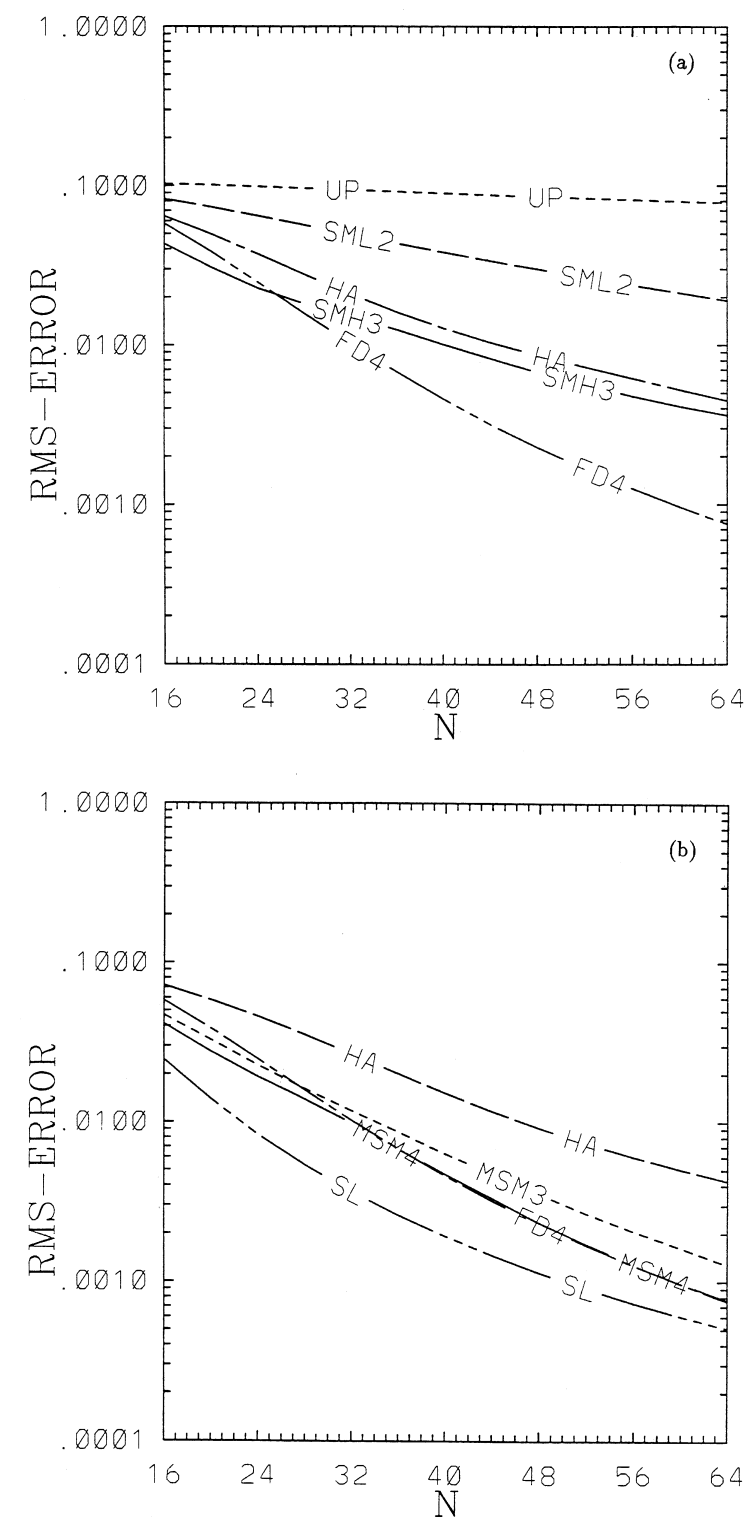

Fig. 3. Root-mean-square error in the numerical solutions of the solid body rotation with Gaussian cone rotation as a function of $N$ after one circulation for (a) the UP,SML2, HA, SMH3 and FD4 methods, and (b) the $H A$, MSM3, MSM4, FD4 and SL methods.

computational time. They are about four times more expensive than the FD4 method. However, when the difference in the stability constraint as shown in Fig. 4 is considered, the $S M H 3$ and $M S M 3$ can be just as efficient as the FD4 method.

Another numerical test concerns the rotation of the slotted cylinder [18,11]. We employ $N=100$ in these calculations. This is a more rigorous test than the gaussian cone test in that both discontinuities and irregular shapes are involved in the advection. In fact, the FD4 

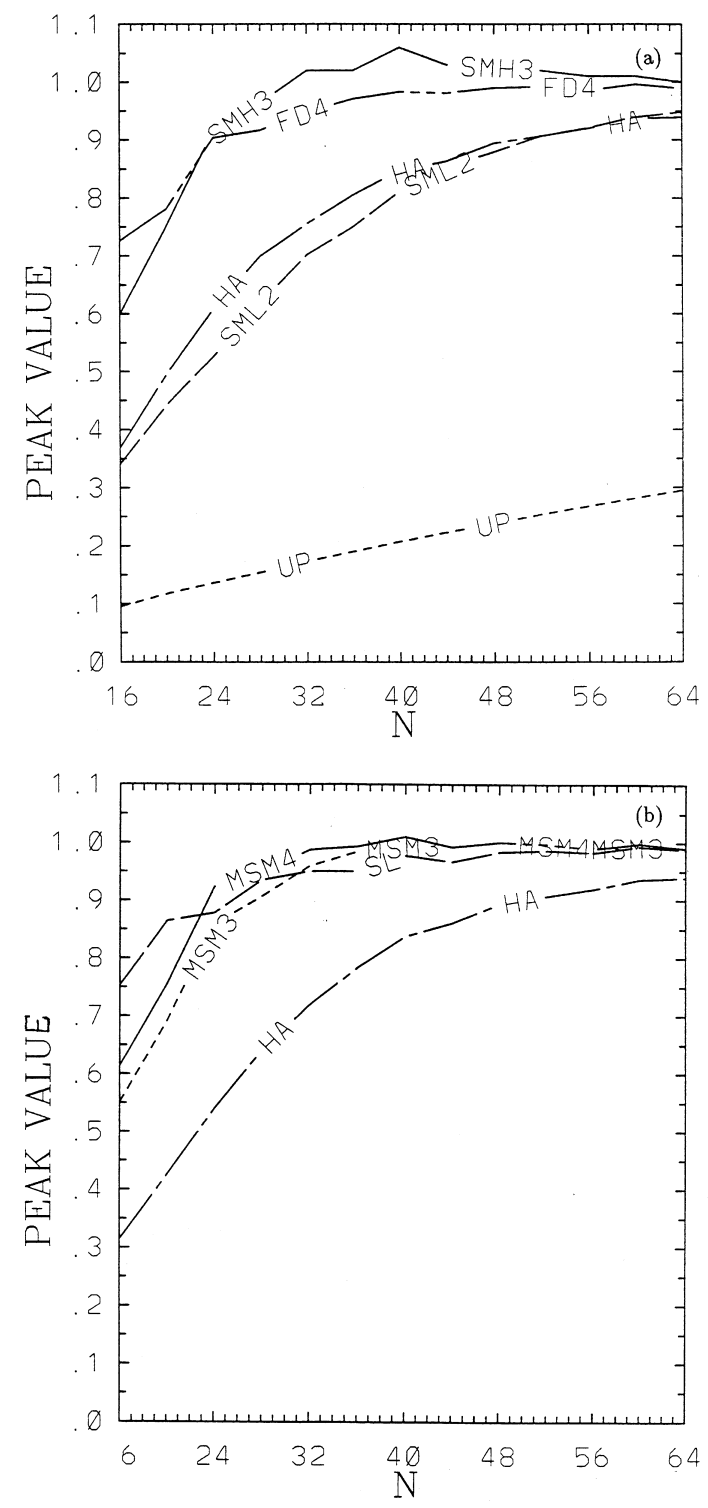

Fig. 4. Peak value in the numerical solutions of the solid body rotation with Gaussian cone rotation as a function of $N$ after one circulation for (a) the UP, SML2, HA, SMH3 and FD4 methods and (b) the HA, MSM3, MSM4 and SL methods.

method yields a very noisy solution and thus is not shown here. The initial profile forms a cylinder with height equal to 1 . The cross sections at $y=0$ and $x=0.5$ as well as the slotted cylinder are shown in Fig. 6a. The numerical solutions for the slotted cylinder after one rotation for the $S L, S M L 2, S M H 3, M S M 3$ and $H A$ are shown in Fig. $6 \mathrm{~b}-\mathrm{f}$, respectively. The proposed MSM3 method compares favorably with the other methods. Note that the SML2 

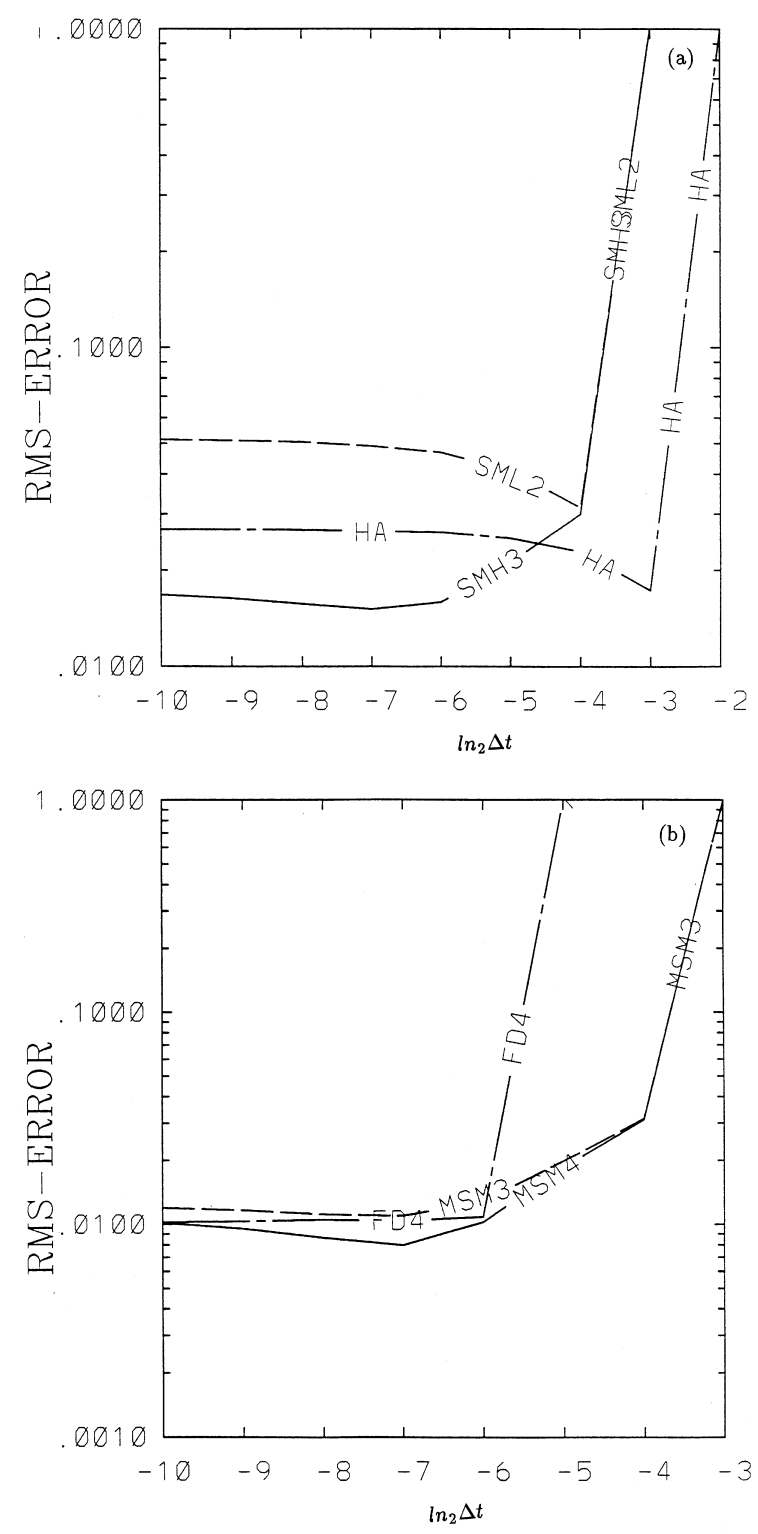

Fig. 5. Root-mean-square error in the numerical solutions of the solid body rotation with Gaussian cone rotation as a function of time step $\ln _{2} \Delta t$ after one circulation for (a) the $S M L 2, H A$ and $S M H 3$ methods, and (b) the MSM3, MSM4 and FD4 methods.

and $H A$ methods yield solutions that are fairly smooth, as can be seen in the cross sections $y=0$ and $x=0.5$, while the $S L, S M H 3$ and MSM3 yield solutions with fine structures. The $S L$ method produces negative regions in the solution. There are no negative regions produced by the $S M H 3$ and MSM3 schemes. Several authors have proposed shape preserving to remove the negative regions in the $S L$ method (see [11] for example). It is beyond the scope of the present paper to give a detailed comparison with the $S L$ methods. 


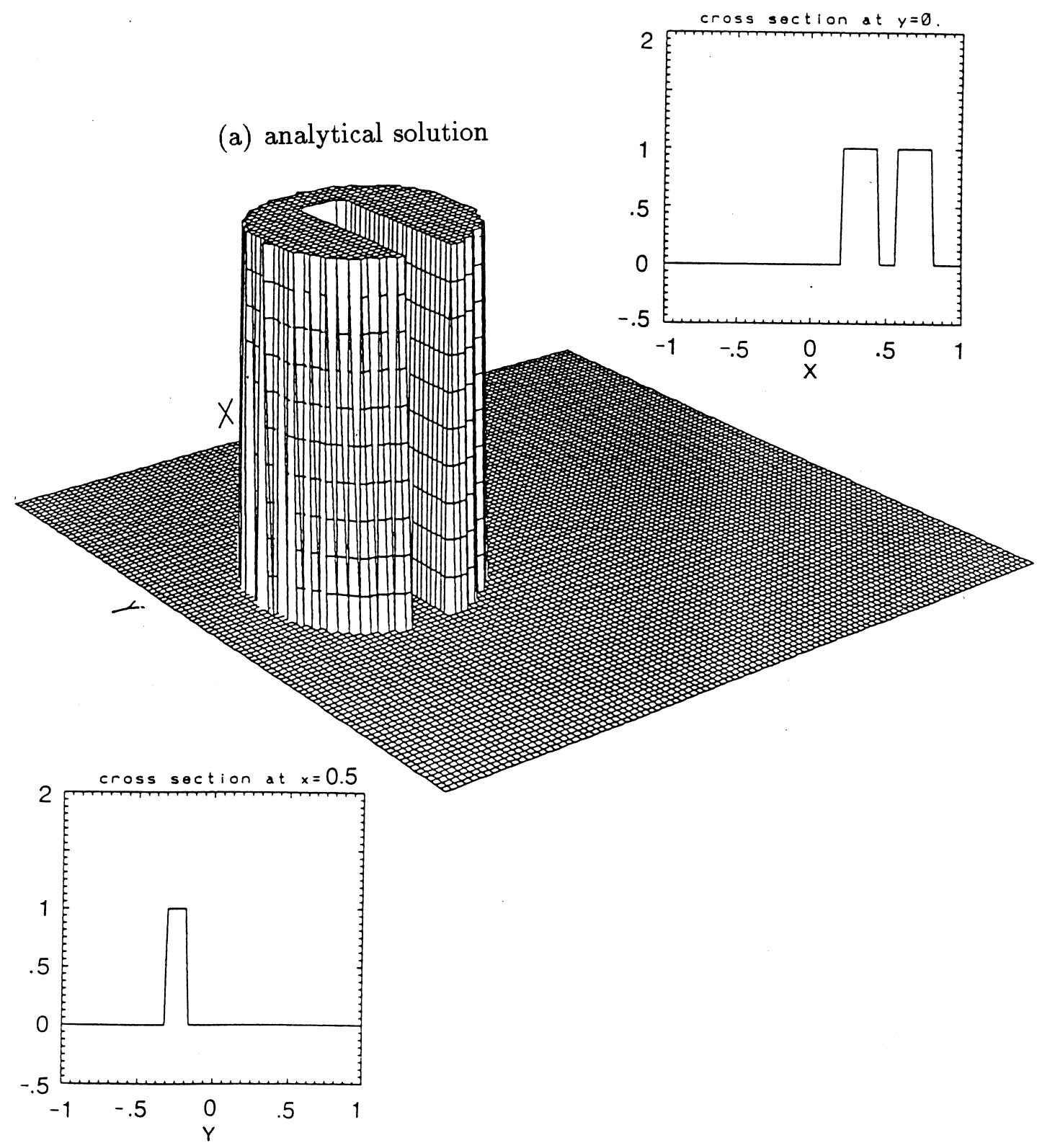

Fig. 6. Numerical solutions of the solid body rotation with slotted cylinder after one circulation with $N=100$ for (a) the analytical solution, (b) the $S L$ method with Courant number equal to 1, (c) the $S M L 2$ method, (d) the $S M H 3$ method, (e) the $M S M 3$ method and (f) the $H A$ method. 


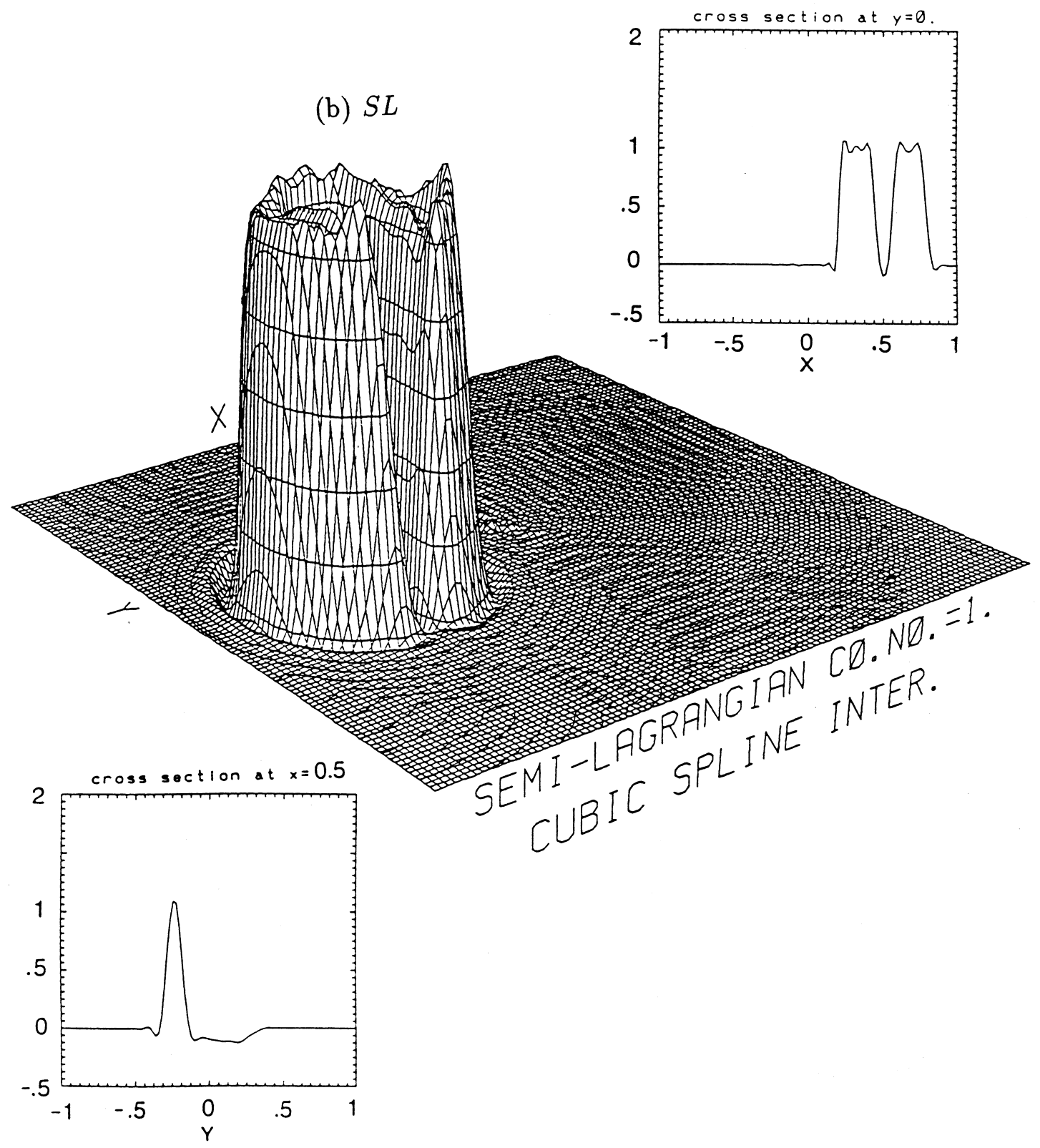

Fig. 6 (b) 


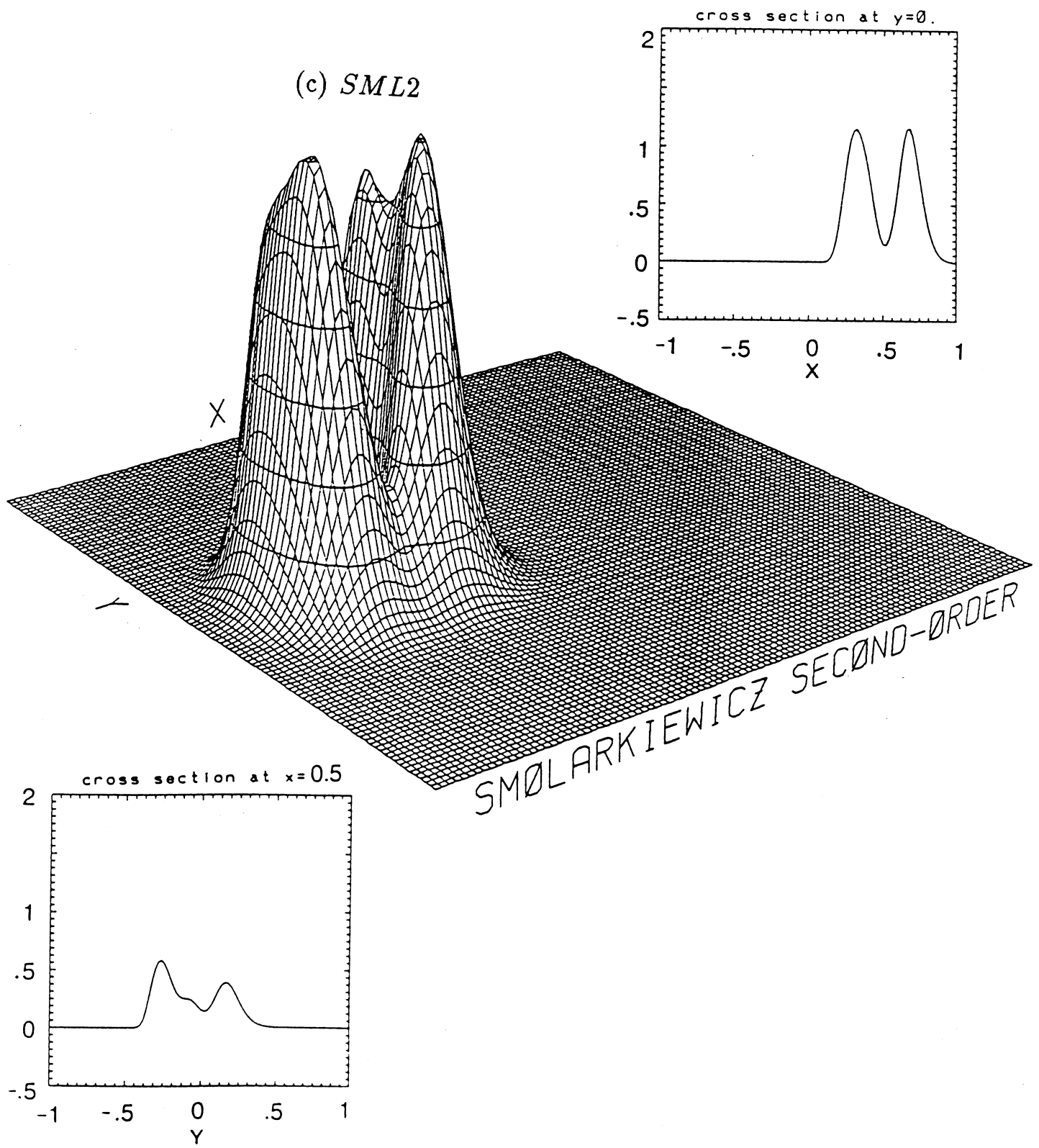

Fig. 6 (c) 


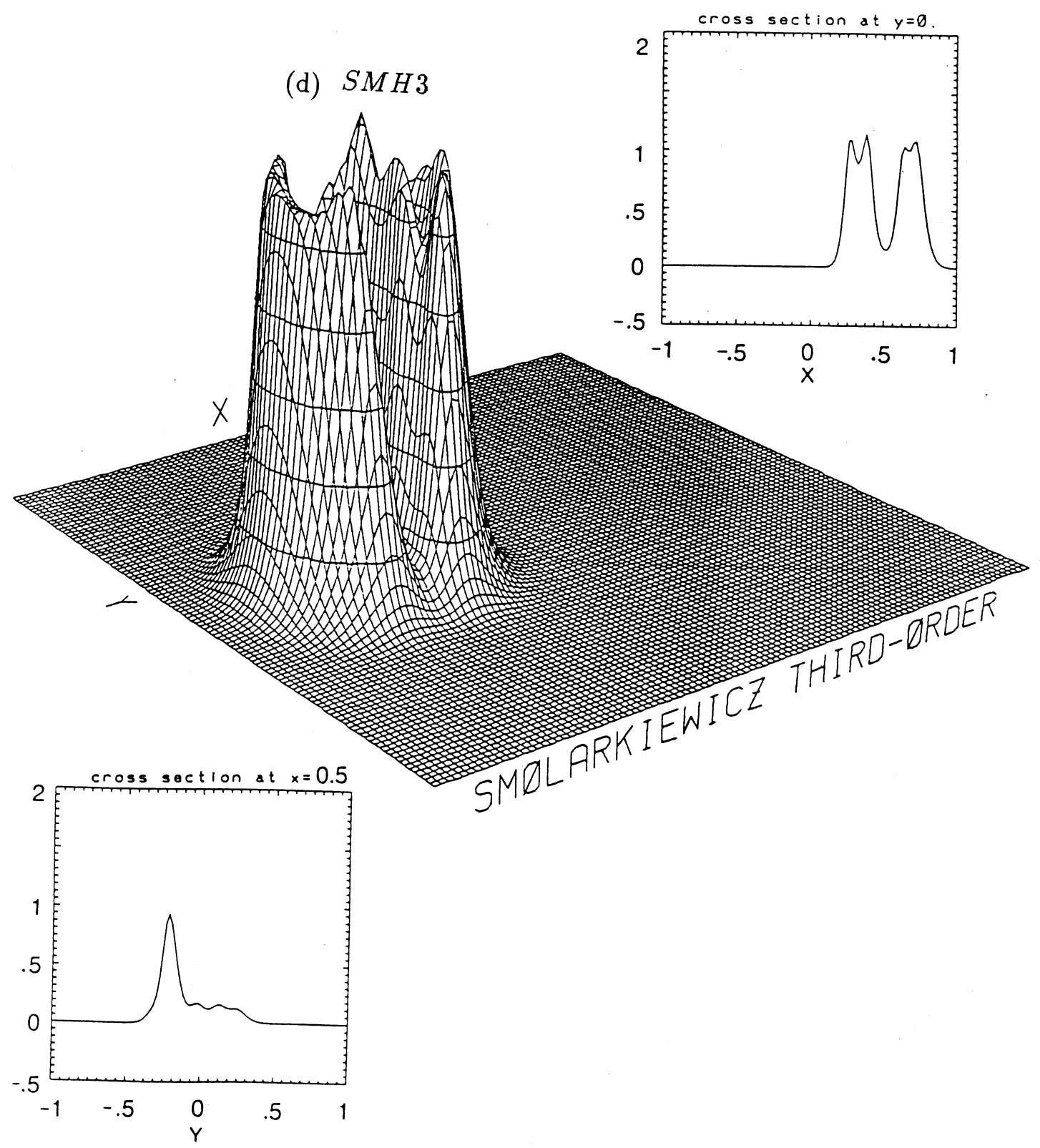

Fig. 6 (d) 
(e) $M S M 3$

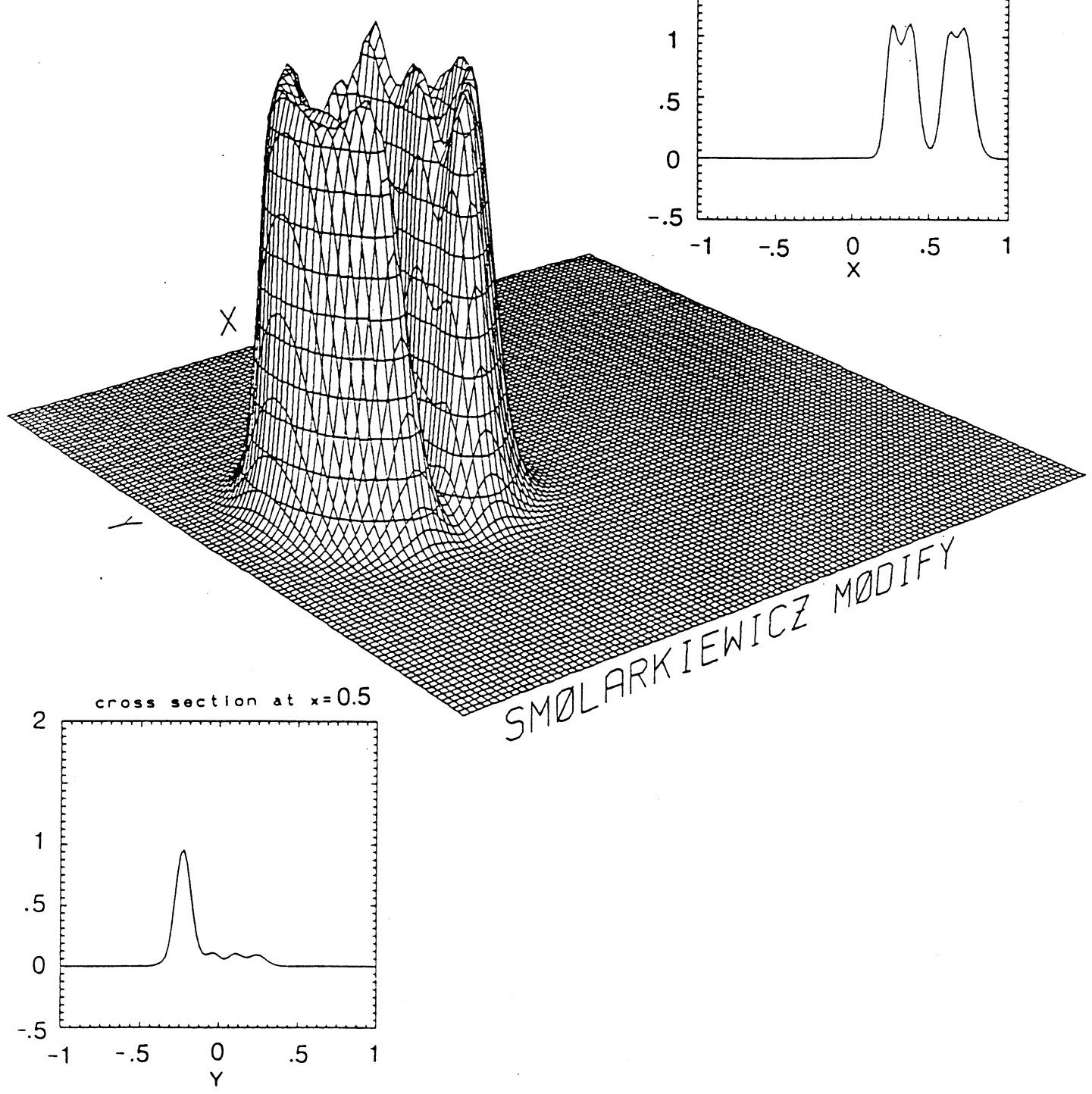

Fig. 6 (e) 
(f) $H A$

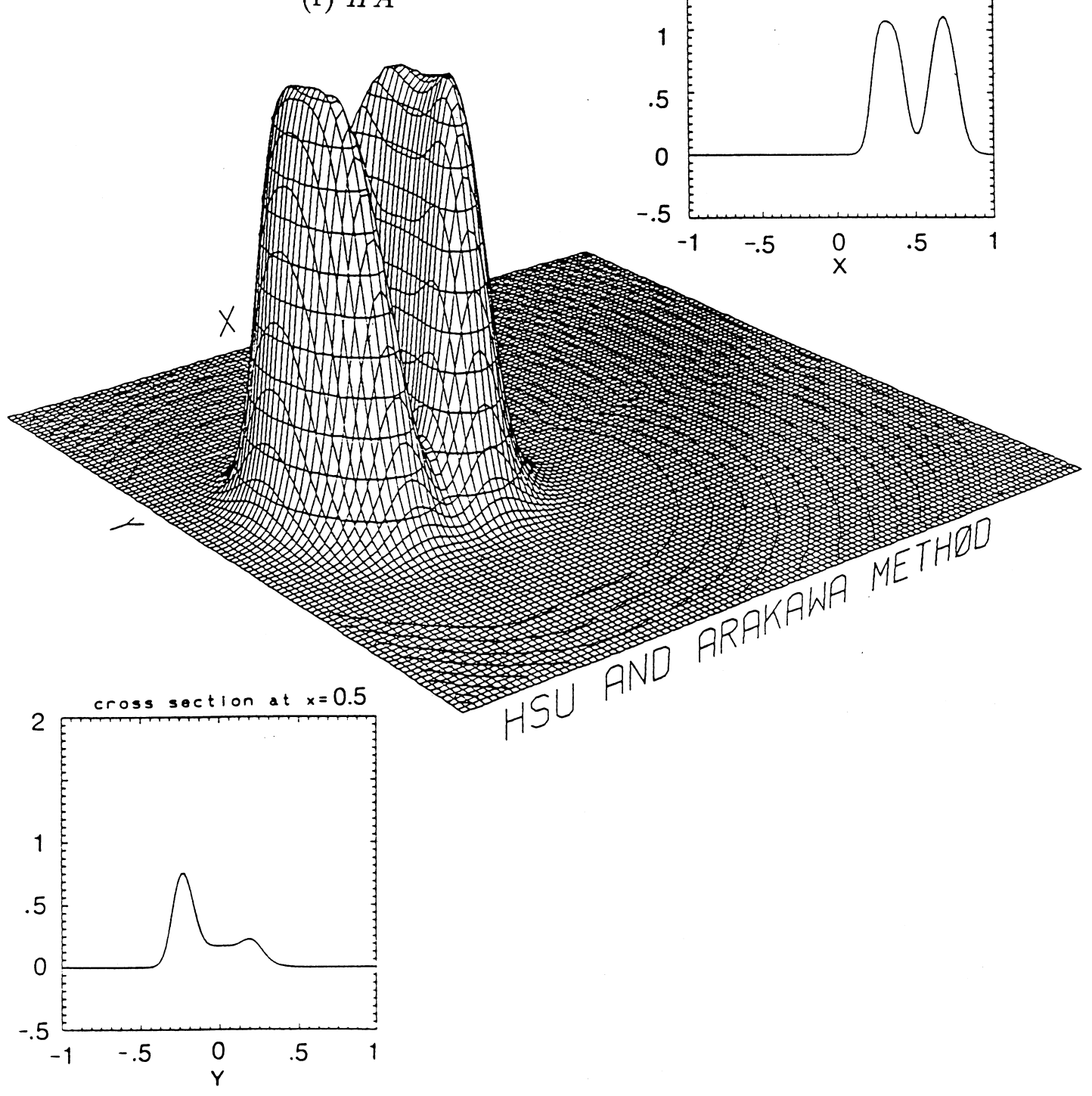

Fig. 6 (f) 


\section{Concluding remarks}

A modified Smolarkiewicz positive definite scheme is proposed, and the stability and consistency conditions are discussed. The computational cost of the proposed scheme is the same as the third-order Smolarkiewicz method. The convergence rate, stability, accuracy and peak value maintenance of the proposed method are demonstrated numerically. Comparative studies of the modified method with other methods are performed and it is found that the modified method can be fourth-order accurate in the low Courant number situation. The stability condition of the proposed method is the same as for the original Smolarkiewicz method. Overall performance of the proposed method compares favorably with the semiLagrangian and fourth-order finite difference schemes. Other tests with the advection of a slotted cylinder also yield forecasts which compare well with forecasts from the high-order (third-order accurate) Smolarkiewicz method and the semi-Lagrangian method, while the fourth-order finite difference scheme is very noisy.

Finally we note that the majority of papers in computational fluid dynamics refer the term "high-order" merely to emphasize compensation of selected high-order terms in the truncation. Genuine high-order accuracy requires compensating all the terms that are of the same order. The 'genuine' third-order scheme of Margolin and Smolarkiewicz [17] has this remarkable property, at least for uniform advection. On the other hand, meteorological models involve time integration of dynamical variables and passive scalars such as moisture field. The dynamical variables and passive scalar are used for application of sophisticated and computationally expensive parameterization schemes. Since the dynamical variables often have fourth-order accuracy, the unmatched accuracy or convergence rate for the water vapor field may yield an undesired feedback to the overall model accuracy. The results from meteorological models are very sensitive to the accuracy of the water vapor field. In view that the time step in meteorological models without the semi-implicit method is limited by the stability constraint, the proposed fourth-order accurate Smolarkiewicz method in the low Courant number situation may be of great potential for handling passive scalar advection.

\section{Acknowledgements}

This work is supported by the grant NSC87-2111-M002-005 AP1 from Taiwan and NSF grant ATM-9525755.

\section{References}

[1] Rood RB. Numerical advection algorithms and their role in atmospheric transport and chemistry models. Rev. Geophys. 1987;25:71-100.

[2] Boris JP, Book DL. Flux-corrected transport. I: SHASTA, a fluid transport a algorithm that works. J. Comput. Phys. 1973;11:38-69.

[3] Zalesak ST. Fully multidimensional flux-corrected transport algorithms for fluids. J. Comput. Phys. 1979;31:335-62. 
[4] Tremback CJ, Powell J, Cotton WR, Pielke RA. The forward-in-time upstream advection scheme: extension to higher orders. Mon. Wea. Rev. 1987;115:540-555.

[5] Hsu Y-J, Arakawa A. Numerical modeling of the atmosphere with an isentropic vertical coordinate. Mon. Wea. Rev. 1990;118:1933-59.

[6] Takacs LL. A two-step scheme for the advection equation with minimized dissipation and dispersion errors. Mon. Wea. Rev. 1985;113:1050-65.

[7] Williamson DL, Rasch PJ. Two-dimensional semi-Lagrangian transport with shape-preserving interpolation. Mon. Wea. Rev. 1989;117:102-29.

[8] Bott A. A positive definite advection scheme obtained by nonlinear renormalization of the advective fluxes. Mon. Wea. Rev. 1989;117:1006-1015.

[9] Bott A. Monotone flux limitation in the area-preservation flux form advection algorithm. Mon. Wea. Rev. 1992;120:2592-2602.

[10] Bott A. The monotone area-preserving flux-form advection algorithm: reducing the time-splitting error in twodimensional flow fields. Mon. Wea. Rev. 1995;121:2637-2641.

[11] Holnicki P. A shape-preserving interpolation:applications to semi-Lagrangian advection. Mon. Wea. Rev. 1995; 123:862-870.

[12] Smolarkiewicz PK. A simple positive definite advection scheme with small implicit diffusion. Mon. Wea. Rev. 1983;111:479-86.

[13] Smolarkiewicz PK. A fully multidimensional positive definite advection transport algorithm with small implicit diffusion. J. Comput. Phys. 1984;54:325-62.

[14] Smolarkiewicz PK, Clark TL. The multidimensional positive definite advection transport algorithm: further development and applications. J. Comput. Phys. 1986;67:396-438.

[15] Smolarkiewicz PK, Grabowski WW. The multidimensional positive definite advection transport algorithm: nonoscillatory option. J. Comput. Phys. 1990;86:355-75.

[16] Smolarkiewicz PK, Rasch PJ. Monotone advection on the sphere: an Eulerian versus semi-Lagrangian approach. J. Atmos. Sci. 1991;48:793-810.

[17] Margolin L, Smolarkiewicz PK. Antidiffusive velocities for multipass donor cell advection. SIAM J. Sci. Comput. 1998 (in press).

[18] Bermejo R, Staniforth A. The conversion of semi-Lagrangian advection schemes to quasi-monotone schemes. Mon. Wea. Rev. 1992;120:2622-2632. 